\title{
Tests that may be overused or misused in cardiology: The Choosing Wisely ${ }^{\circledR}$ campaign
}

\author{
George A. Beller, MD
}

The American College of Cardiology (ACC) released a list of "Five Things Physicians and Patients Should Question', in cardiology as part of Choosing Wisely ${ }^{\circledR}$ which is an initiative of the American Board of Internal Medicine (ABIM) Foundation in partnership with Consumer Reports. These were among 45 common tests and procedures recommended by nine medical specialty boards that should be performed less often. Patients were urged by these boards to question these services if and when they are offered.

The American College of Cardiology's list ${ }^{1}$ of five recommendations included four that were related to cardiac imaging, particularly, stress tests or advanced noninvasive imaging. The first was that stress tests or advanced noninvasive imaging should not be performed if there are no symptoms of heart disease or high-risk factors like diabetes or peripheral arterial disease are not present. The second stated that cardiac imaging tests, particularly stress tests, should not be given as part of a routine annual follow up in patients who have had no change in signs or symptoms. The third recommendation was that cardiac imaging tests should not be given before performing low-risk surgery that is not related to heart disease. In addition, a fourth recommendation cited that echocardiograms should not be used as routine follow-up care in adults with mild heart valve disease, who have had no change in signs and symptoms. These recommendations by the ACC are entirely consistent with ACC/AHA Practice guidelines and the ACC's Appropriate Use Criteria (AUC). In addition to contributing to the rising costs of cardiovascular imaging, ordering stress myocardial perfusion imaging (MPI) tests for inappropriate indications adds to the radiation exposure of patients. ${ }^{2}$ Downstream testing for equivocal or false positive test results adds to both the cost and radiation exposure if cardiac catheterization is performed. The positive predictive accuracy of a noninvasive imaging

From the University of Virginia, Charlottesville, VA.

Reprint requests: George A. Beller, MD, University of Virginia, Charlottesville, VA; gbeller@virginia.edu.

J Nucl Cardiol 2012;19:401-3.

$1071-3581 / \$ 34.00$

Copyright (C) 2012 American Society of Nuclear Cardiology.

doi:10.1007/s12350-012-9569-y test in a population with a low pretest likelihood of CAD may be problematic and may contribute to the rather high rate of normal coronary angiograms seen in some cardiac catheterization laboratories. $^{3}$

The cardiology imaging community has already been promulgating reducing the rate of inappropriate cardiac imaging procedures while documenting precisely the rate and type of inappropriate imaging tests being ordered. The most frequently documented inappropriate indications include those cited above by the ACC, ${ }^{1}$ which should be performed less often. In one multicenter trial, ${ }^{4}$ the rate of inappropriate cardiac imaging SPECT studies was $15 \%$. The most frequent inappropriate indications were detection of CAD in asymptomatic patients with low CAD risk (44.5\%), testing asymptomatic patients after revascularization $<2$ years after a PCI who had symptoms before the prior PCI $(23.8 \%)$ and evaluation of chest pain in patients with a low probability of CAD, an interpretable baseline ECG, and being able to exercise (16.1\%). More inappropriate tests are ordered by non-cardiologists and, in one recent study, the majority of inappropriate or uncertain studies were ordered in women. ${ }^{5}$ In this study, women had $68 \%$ of inappropriate studies, and $82 \%$ of uncertain studies. In fact, the value of adding exercise MPI to the standard ECG exercise treadmill test in symptomatic women with a normal resting ECG and a Duke Activity Status Index of $\geq 5$ METS has been questioned. ${ }^{6}$ In the WOMEN trial of low-risk exercising women, exercise ECG testing vs exercise MPI yielded similar outcomes after two years of follow-up. In our own study, patients at an intermediate pretest risk for CAD who achieved target heart rate adjusted for age and $\geq 10$ METS of workload on exercise treadmill testing, had a very low prevalence $(0.4 \%)$ of significant ischemia ( $>10 \%$ of the left ventricle), and a $0.1 \%$ annual rate of future cardiac death and a $0.3 \%$ annual rate of nonfatal infarction. ${ }^{7,8}$ The added value of MPI to standard ECG stress testing is questionable in such patients with high exercise tolerance. Interestingly, patients who achieved $\geq 10$ METS comprised about one-third of all the patients referred to the nuclear cardiology laboratory for exercise imaging over a one-year period. The cost savings extrapolated to the entire United States if exercise treadmill testing is performed first without MPI are immense. The results of our study and the WOMEN trial 
suggest that a protocol of provisional MPI should be tested in a clinical research study in which patients at low-to-intermediate pretest probability of CAD, a normal resting ECG, and good functional capacity not be injected with tracer if they achieve a workload of $\geq 10$ METS with no ST segment depression.

The attention being directed at reducing the volume of cardiac imaging procedures that are inappropriate is occurring at a time when new imaging indications are emerging that may significantly impact on patient management and adherence to effective guideline-based therapy. A good example is CT coronary artery calcium (CAC) imaging which is relatively inexpensive and associated with very low radiation exposure. Many insurance companies still do not cover CT CAC imaging, although it received a Class IIA recommendation in the ACC/AHA guidelines for risk assessment in asymptomatic adults at intermediate risk by clinical evaluation. The EISNER trial randomized patients to groups that either did or did not have CAC scanning before risk factor counseling. ${ }^{9}$ The primary endpoint was a 4-year change in CAD risk factors and the Framingham Risk Score. Compared to the no-scan group, the group that had CAC imaging before counseling had a more favorable net change in systolic blood pressure, LDL-cholesterol levels, and waist circumference, and the higher the baseline CAC score, the greater the improvement in these CAD risk factors. Interestingly, the study showed no increase in downstream testing in the group randomized to CT CAC imaging.

At the March 2012 Annual Scientific Sessions of the ACC, Kalia et al ${ }^{10,11}$ reported the results of two studies in which patients were shown their CAC scans. The investigators reported that, adjusted for age, gender, and race, patients who had any CAC were twice as likely to be compliant with their statin regimen, and patients who had severe CAC were 2.6 times as likely to be compliant with statin therapy, compared with patients with a CAC score of zero. A second study from the same group showed that in patients who saw their own CT CAC scans, and had no evidence of CAC, only $20 \%$ lost weight during follow-up. In contrast, $40 \%$ of patients with CAC scores of over 400 lost weight. Patients with a CAC score of over 400 were 3.3 times as likely as those with no CAC, to lose weight. Thus, the results of both the EISNER trial and the studies by Kalia et al found that determination of CAC, and showing the calcium in the coronary arteries to patients, improves risk-factor modification, promotes weight loss, and results in greater adherence to statin therapy. These beneficial effects should improve outcomes based on other studies showing that lowering of an abnormally elevated LDL-cholesterol, treating hypertension, and losing weight lowers the risk of future cardiac events. Cost effectiveness still needs to be demonstrated in future studies of the influence of CT CAC scanning on patient management.

Thus, the initiative by the ACC as part of the Choosing Wisely ${ }^{\circledR}$ campaign to reduce the number of inappropriate cardiac imaging tests needs to be balanced by a willingness to approve new imaging tests if they impact favorably on clinical management and outcomes. The example given for CT CAC imaging is just one emerging application of imaging in patients with cardiovascular disease. Another is CT coronary angiography performed in low-to-intermediate risk patients presenting in the emergency department with symptoms suggestive of CAD or an acute coronary syndrome. As evidence from clinical trials accumulates, the ACC and other organizations should objectively determine clinical indications and appropriateness with consideration of safety and cost effectiveness. Clinicians ordering imaging tests need to be educated with respect to when imaging tests should not be performed as well as when they do provide valuable information for clinical care. Organizations like the ACC and the American Society of Nuclear Cardiology remain at the forefront for advocating quality and guideline-based recommendations for indications for imaging tests. We have not only an obligation to support the Choosing Wisely ${ }^{\circledR}$ campaign but also the responsibility to advocate for new and existing imaging technologies that improve patient care.

\section{References}

1. http://www.cardiosource.org/News-Media/Publications/CardiologyMagazine/choosing-wisely.aspx. Accessed 6 April 2012.

2. Einstein AJ. Effects of radiation exposure from cardiac imaging: How good are the data? J Am Coll Cardiol 2012;59:553-65.

3. Douglas PS, Patel MR, Bailey SR, et al. Hospital variability in the rate of finding obstructive coronary artery disease at elective diagnostic coronary angiography. J Am Coll Cardiol 2011;58: 801-9.

4. Hendel RC, Cerqueira M, Douglas PS, et al. A multicenter assessment of the use of single-photon emission computed tomography myocardial perfusion imaging with appropriateness criteria. J Am Coll Cardiol 2010;55:156-62.

5. Gupta A, Tsiaras SV, Dunsiger SI. Tilkemeier. Gender disparity and the appropriateness of myocardial perfusion imaging. J Nucl Cardiol 2011;18:588-94.

6. Shaw LJ, Mieres JH, Hendel RH, et al. Comparative effectiveness of exercise electrocardiography with or without myocardial perfusion single photon emission computed tomography in women with suspected coronary artery disease: Results from the What Is the Optimal Method for Ischemia Evaluation in Women (WOMEN) Trial. Circulation 2011;124:1239-49.

7. Bourque JM, Holland BH, Watson DD, Beller GA. Achieving an exercise workload of $\geq 10$ metabolic equivalents predicts a very low risk of inducible ischemia: Does myocardial perfusion imaging have a role? J Am Coll Cardiol 2009;54:538-45. 
8. Bourque JM, Charloton GT, Holland BJ, Belyea CM, Watson DD, Beller GA. Prognosis in patients achieving $\geq 10$ METS on exercise stress testing: Was SPECT imaging useful? J Nucl Cardiol 2011;18:230-7.

9. Rozanski A, Gransar H, Shaw LJ, Kim J, Miranda-Peats L, Wong $\mathrm{ND}$, et al. Impact of coronary artery calcium scanning in coronary risk factors and downstream testing. The EISNER (Early Identification of Subclinical Atherosclerosis by Noninvasive Imaging
Research) prospective randomized trial. J Am Coll Cardiol 2011;57:1622-7.

10. Kalia N, Li D, Budoff M. Statin therapy adherence after visualizing coronary calcium by cardiac computed tomography. J Am Coll Cardiol 2012;59:E1188.

11. Kalia N, Li D, Budoff M. Motivational effect on weight loss after visualizing coronary calcium by cardiac computed tomography. $\mathrm{J}$ Am Coll Cardiol 2012;59:E1189. 